\title{
THE NULL DIVERGENCE FACTOR
}

\author{
J. Chavarriga** H. Giacomini and J. Giné*
}

\begin{abstract}
Let $(P, Q)$ be a $C^{1}$ vector field defined in a open subset $U \subset R^{2}$. We call a null divergence factor a $C^{1}$ solution $V(x, y)$ of the equation $P \frac{\partial V}{\partial x}+Q \frac{\partial V}{\partial y}=\left(\frac{\partial P}{\partial x}+\frac{\partial Q}{\partial y}\right) V$. In previous works it has been shown that this function plays a fundamental role in the problem of the center and in the determination of the limit cycles. In this paper we show how to construct systems with a given null divergence factor. The method presented in this paper is a generalization of the classical Darboux method to generate integrable systems.
\end{abstract}

\section{Introduction}

We consider in this paper two-dimensional autonomous systems of differential equations of the form

$$
\dot{x}=P(x, y), \quad \dot{y}=Q(x, y), \quad \cdot=\frac{d}{d t},
$$

with $P(x, y), Q(x, y) \in C^{1}(E)$ and where $E$ is an open subset of $R^{2}$.

The two fundamental problems of the qualitative theory of system (1) are the problem of the center and the determination of the number of limit cycles and their location in phase space.

In recent works it has been shown that a unified method can be used to study these problems $[\mathbf{1}],[\mathbf{2}],[\mathbf{3}],[\mathbf{4}],[\mathbf{5}],[\mathbf{9}],[\mathbf{1 0}],[\mathbf{1 1}]$, and $[\mathbf{1 2}]$.

The method is based on the determination of a function $V(x, y) \in$ $C^{1}(E)$ that satisfies the equation

$$
P \frac{\partial V}{\partial x}+Q \frac{\partial V}{\partial y}=\left(\frac{\partial P}{\partial x}+\frac{\partial Q}{\partial y}\right) V
$$

*Research partially supported by a University of Lleida Project 93-3. 
Let us consider first the center problem for polynomial systems of the form:

$$
\dot{x}=-y+X(x, y), \quad \dot{y}=x+Y(x, y),
$$

where $X(x, y)$ and $Y(x, y)$ are polynomials without independent and linear terms.

The problem of the center consists in giving necessary and sufficient conditions on the coefficients of $X(x, y)$ and $Y(x, y)$ in order to have a continuous family of periodic orbits in a certain neighbourhood of the origin. If for system (3) we can find a solution $V(x, y) \in C^{1}(E)$ of (2) that is not zero at the origin, then we can obtain a first integral of (3) well defined in a neighbourhood of the origin, because $M(x, y)=\frac{1}{V(x, y)}$ is an integrating factor of the system. In that case, the origin will be a center of $(3)$.

For many systems of type (3) having a center at the origin, it has been shown in [1], [2], [4], [5] and [11] that the function $V(x, y)$ has very simple properties, being very often a polynomial. By contrary, the first integral is, in general, a complicated expression that can not be written in terms of elementary functions.

In particular, when in system (3) $X$ and $Y$ are both quadratic or cubic homogeneous polynomials the function $V(x, y)$ is a polynomial for all center cases (see [1] ).

In the general case, i.e. for system (1), it has been shown in $[\mathbf{3}],[\mathbf{9}]$, $[\mathbf{1 0}]$ and $[\mathbf{1 2}]$ that any solution of (2) plays a fundamental role in the determination of the limit cycles of the system. Esentially, $V(x, y)$ must vanish on all limit cycles of (1) (for a precise formulation of these results see $[\mathbf{9}])$.

In this paper we present a method which enables us to generate (or construct) systems of type (1) with a known function $V(x, y)$. In this way, for all systems generated with this method, we know at once all limit cycles and all centers. These results are presented in sections 2 and 3 .

\section{Construction of systems with a known function $V(x, y)$}

We generalise in this section the classical Darboux method for constructing integrable systems [8].

Based in the Darboux method the next result follows easily from Christopher results (see $[\mathbf{6}]$ and $[\mathbf{1 3}]$ ). 
The vector field defined by:

(4)

$$
P=\sum_{i=1}^{n} a_{i}\left(\prod_{\substack{j=1 \\ j \neq i}}^{n} f_{j}(x, y)\right) \frac{\partial f_{i}(x, y)}{\partial y}
$$

$$
Q=-\sum_{i=1}^{n} a_{i}\left(\prod_{\substack{j=1 \\ j \neq i}}^{n} f_{j}(x, y)\right) \frac{\partial f_{i}(x, y)}{\partial x}
$$

where $f_{j}(x, y)$ (with $j=1, \ldots, n$ ) are arbitrary $C^{2}$ functions, $n \in N$ and the $a_{i}$ are arbitrary real parameters, has a first integral given by

$$
I(x, y)=\prod_{i=1}^{n} f_{i}^{a_{i}}
$$

and an integrating factor

$$
M(x, y)=\prod_{i=1}^{n} f_{i}^{-1}
$$

As it is well known, a system with a Darboux type first integral (5) can not have limit cycles in the domain of definition of the Darboux first integral. In particular if $a_{i}$ are rational numbers system (4) can not have limit cycles.

Instead of giving a vector field with a known first integral, we construct a system with a known function $V(x, y)$ as follows:

Proposition 1. Let $\left(P_{i}, Q_{i}\right)$, with $i=1, \ldots, n$, be $C^{1}$ vector fields defined in an open subset $U \subset R^{2}$, which have $C^{2}$ null divergence factors $V_{i}(x, y)$, i.e.

$$
P_{i} \frac{\partial V_{i}}{\partial x}+Q_{i} \frac{\partial V_{i}}{\partial y}=\left(\frac{\partial P_{i}}{\partial x}+\frac{\partial Q_{i}}{\partial y}\right) V_{i}
$$

with $i=1, \ldots, n$. 
Then, the vector field

(8)

$$
P=\lambda_{0} \frac{\partial\left(\prod_{i=1}^{n} V_{i}(x, y)\right)}{\partial y}+\sum_{i=1}^{n} \lambda_{i}\left(\prod_{\substack{j=1 \\ j \neq i}}^{n} V_{j}(x, y)\right) P_{i}(x, y)
$$

$$
Q=-\lambda_{0} \frac{\partial\left(\prod_{i=1}^{n} V_{i}(x, y)\right)}{\partial x}+\sum_{i=1}^{n} \lambda_{i}\left(\prod_{\substack{j=1 \\ j \neq i}}^{n} V_{j}(x, y)\right) Q_{i}(x, y)
$$

has a null divergence factor $V(x, y)$ given by

$$
V(x, y)=\prod_{i=1}^{n} V_{i}(x, y)
$$

Proof: The proof is straight-forward. The first integral of (8) can be calculated from the integrating factor $M(x, y)=\frac{1}{V(x, y)}$.

In general, this first integral will not be defined in the whole domain of the definition of the differential system and it is possible for system (8) to have limit cycles.

It is clear that (4) is a particular case of (8), with $V_{i}(x, y)=f_{i}(x, y)$, $P_{i}(x, y)=\frac{\partial f_{i}}{\partial y}, Q_{i}(x, y)=-\frac{\partial f_{i}}{\partial x}, \lambda_{i}=a_{i}$ and $\lambda_{0}=0$.

In (4) all vector fields $\left(P_{i}, Q_{i}\right)$ used to generate the system $(P, Q)$ are Hamiltonian, while in (8) they are arbitrary. This is the key point of our generalization of the classical Darboux method.

It is interesting to note that well-known systems can be constructed from (8) by using linear systems and Hamiltonian systems $\left(P_{i}, Q_{i}\right)$. Let us consider several examples:

Example 1. In [14], a quartic system with one center and one limit cycle has been studied. The system is:

$$
\begin{aligned}
P= & -2 y\left(x^{2}+y^{2}\right)(x-2)+(x-y)\left(x^{2}+2 y^{2}-1\right)(x-2), \\
Q= & x\left(x^{2}+y^{2}\right)(x-2)+(x+y)\left(x^{2}+2 y^{2}-1\right)(x-2) \\
& -\frac{7}{10}\left(x^{2}+2 y^{2}-1\right)\left(x^{2}+y^{2}\right) .
\end{aligned}
$$


The null divergence factor of this system is:

$$
V(x, y)=(x-2)\left(x^{2}+y^{2}\right)\left(x^{2}+2 y^{2}-1\right) .
$$

The limit cycle of (10) is the ellipse $x^{2}+2 y^{2}-1=0$ and the center is located at the point $(3,1)$. This system can be generated by using (8), as follows:

$$
\begin{aligned}
& P=P_{1} V_{2} V_{3}+P_{2} V_{1} V_{3}+P_{3} V_{1} V_{2}, \\
& Q=Q_{1} V_{2} V_{3}+Q_{2} V_{1} V_{3}+Q_{3} V_{1} V_{2},
\end{aligned}
$$

where

$$
\begin{aligned}
(13) & \left(P_{2}, Q_{2}\right)=\left(0,-\frac{7}{10}\right), \quad \text { with } \quad V_{2}(x, y)=(x-2) \quad \text { and } \\
\left(P_{3}, Q_{3}\right) & =(x-y, x+y), \quad \text { with } \quad V_{3}(x, y)=\left(x^{2}+y^{2}\right) .
\end{aligned}
$$

For this case we have $n=3, \lambda_{0}=0, \lambda_{1}=\lambda_{2}=\lambda_{3}=1$. Systems $\left(P_{1}, Q_{1}\right)$ and $\left(P_{2}, Q_{2}\right)$ are Hamiltonian. System $\left(P_{3}, Q_{3}\right)$ is a linear non-Hamiltonian vector field.

Let us recall that the null divergence factor of a linear system

$$
\begin{aligned}
& P=a x+b y \\
& Q=c x+d y
\end{aligned}
$$

is given by

$$
V(x, y)=c x^{2}+(d-a) x y-b y^{2}
$$

For a Hamiltonian vector field $P=\frac{\partial H(x, y)}{\partial y}, Q=-\frac{\partial H(x, y)}{\partial x}$, the null divergence factor is $V(x, y)=f(H(x, y))$ where $f$ is an arbitrary function.

Example 2. The cubic system

$$
\begin{aligned}
P= & y+a_{20} x^{2}+a_{11} x y-2 a_{20} y^{2}+a_{20} b_{20} x^{3}+a_{21} x^{2} y \\
& -a_{11} a_{20} x y^{2}+a_{20}^{2} y^{3}, \\
Q= & -x-b_{20} x^{2}-\left(\frac{b_{21}}{b_{20}}-a_{20}\right) x y-b_{21} x^{2} y+\frac{a_{20} b_{21}}{b_{20}} x y^{2},
\end{aligned}
$$


with $b_{20} \neq 0$ and $a_{20} b_{20}+b_{21} \neq 0$, has been studied in [11]. It has a center at the origin and the null divergence factor is:

$$
\begin{aligned}
V(x, y)= & \left(b_{20}+b_{21} y\right)\left(-a_{20} b_{20}\left(a_{20} b_{20}+b_{21}\right) x^{3}\right. \\
& +\left(a_{21} b_{20}-a_{20} b_{21}\right) x^{2}\left(1-a_{20} y\right) \\
& \left.+a_{11} b_{20} x\left(1-a_{20} y\right)^{2}+b_{20}\left(1-a_{20} y\right)^{3}\right) .
\end{aligned}
$$

This system can be expressed as the composition of two systems $\left(P_{1}, Q_{1}\right)$ and $\left(P_{2}, Q_{2}\right)$, as follows:

$$
\begin{aligned}
& P=P_{1} V_{2}+P_{2} V_{1}, \\
& Q=Q_{1} V_{2}+Q_{2} V_{1},
\end{aligned}
$$

where

$$
\begin{aligned}
\left(P_{1}, Q_{1}\right)= & \left(\frac{-1}{a_{20} b_{20}+b_{21}}, 0\right), \quad \text { with } V_{1}=b_{20}+b_{21} y \quad \text { and } \\
\left(P_{2}, Q_{2}\right)= & \left(( a _ { 2 0 } b _ { 2 0 } + b _ { 2 1 } ) ^ { - 1 } \left(1+a_{11} x+\left(a_{20}^{2}+a_{21}\right) x^{2}-2 a_{20} y\right.\right. \\
& \left.\left.-a_{11} a_{20} x y+a_{20}^{2} y^{2}\right), \frac{x}{b_{20}}\left(-1-b_{20} x+a_{20} y\right)\right),
\end{aligned}
$$

with

$$
\begin{aligned}
V_{2}= & -a_{20} b_{20}\left(a_{20} b_{20}+b_{21}\right) x^{3}+\left(a_{21} b_{20}-a_{20} b_{21}\right) x^{2}\left(1-a_{20} y\right) \\
& +a_{11} b_{20} x\left(1-a_{20} y\right)^{2}+b_{20}\left(1-a_{20} y\right)^{3} .
\end{aligned}
$$

System $\left(P_{1}, Q_{1}\right)$ is a constant Hamiltonian vector field and $\left(P_{1}, Q_{1}\right)$ is an integrable quadratic system.

Example 3. The cubic system

$$
\begin{aligned}
& P=y, \\
& Q=-x+k(1-l) x^{2}+k^{2} l x^{3}+a_{2} x y-a_{2} k x^{2} y+k l y^{2}-\frac{k^{2} l^{2}}{1+l} x y^{2},
\end{aligned}
$$

with $l+1 \neq 0$, has been studied in $[\mathbf{7}]$. It has a center at the origin and its null divergence factor is 


$$
V=1+2 k l x+k^{2} l^{2} x^{2}-a_{2} y-a_{2} k l x y-\frac{k^{2} l^{3}}{1+l} y^{2} .
$$

This system can be expressed as the composition of two systems, when $k l(l+1) \neq 0$, as follows:

$$
\begin{aligned}
& P=P_{1} V_{2}+P_{2} V_{1}, \\
& Q=Q_{1} V_{2}+Q_{2} V_{1},
\end{aligned}
$$

with

$$
\begin{array}{ll}
\left(P_{1}, Q_{1}\right)=\left(y, \frac{(1+l)}{k l^{2}}\left(1+k l x-a_{2} y\right)\right), & \text { with } \quad V_{1}=V \quad \text { and } \\
\left(P_{2}, Q_{2}\right)=\left(0, \frac{(-1-l+k l x)}{k l^{2}}\right), & \text { with } \quad V_{2}=1 .
\end{array}
$$

Example 4. The quadratic system

$$
\begin{aligned}
& P=-y-b x^{2}-c x y-d y^{2}, \\
& Q=x+a x^{2}+A x y-a y^{2},
\end{aligned}
$$

has a center at the origin if and only if one of the following conditions is satisfied.

$$
\begin{array}{ll}
\text { (i) } & A-2 b=c+2 a=0, \\
\text { (ii) } & c=a=0 \\
\text { (iii) } & b+d=0 \\
\text { (iv) } & c+2 a=A+3 b+5 d=a^{2}+b d+2 d^{2}=0 .
\end{array}
$$

In all the cases, system (24) can be descomposed in terms of more simple systems. 
The case (i) corresponds to a Hamiltonian system and is a particular case of (8), with $n=1, \lambda_{0}=1$ and $V_{1}(x, y)=H(x, y)$, where $H(x, y)$ is the Hamiltonian of the system.

For the case (ii), with $(A+b)(A+2 b) \neq 0$, the system can be written as

$$
\begin{aligned}
& P=\frac{\partial\left(V_{1} V_{2}\right)}{\partial y}+P_{1} V_{2}+P_{2} V_{1}, \\
& Q=-\frac{\partial\left(V_{1} V_{2}\right)}{\partial x}+Q_{1} V_{2}+Q_{2} V_{1},
\end{aligned}
$$

where

$$
\begin{aligned}
\left(P_{1}, Q_{1}\right)= & \left(-\frac{\left(1+A^{3}+3 A^{2} b+2 A b^{2}\right)}{(A+b)(A+2 b)}, 0\right), \quad \text { with } \quad V_{1}=1+A y, \text { and } \\
\left(P_{2}, Q_{2}\right)= & \left(\frac{\left(1+2 A^{2} b+6 A b^{2}+4 b^{3}\right)}{(A+b)(A+2 b)}(-A-b+d-A d y-2 b d y),\right. \\
& \left.\left(1+2 A^{2} b+6 A b^{2}+4 b^{3}\right) x\right),
\end{aligned}
$$

with

$$
V_{2}=-A-b+d+b(A+b)(A+2 b) x^{2}+2 b(A+b-d) y+b d(A+2 b) y^{2} .
$$

For the case (iii), without loss of generality, we can take $a=0$. In this case, system (24) can be decomposed as follows:

$$
\begin{aligned}
& P=\frac{\partial\left(V_{1} V_{2}\right)}{\partial y}+P_{1} V_{2}+P_{2} V_{1}, \\
& Q=-\frac{\partial\left(V_{1} V_{2}\right)}{\partial x}+Q_{1} V_{2}+Q_{2} V_{1},
\end{aligned}
$$

where $A+b \neq 0$ and 
(29)

$$
\begin{aligned}
\left(P_{1}, Q_{1}\right)= & \left(\frac{1-A^{2}-A b}{A+b}, 0\right), \quad \text { with } \quad V_{1}=1+A y \quad \text { and } \\
\left(P_{2}, Q_{2}\right)= & \left(\left(2 A b+2 b^{2}-1+\left(A c b+c b^{2}-c\right) x+\left(b-2 A b^{2}-2 b^{3}\right) y\right)\right. \\
& \left.(A+b)^{-1}, c+x-2 A b x-2 b^{2} x-c b y\right),
\end{aligned}
$$

with

$$
V_{2}=(1-b y)^{2}+c(1-b y) x-b(A+b) x^{2} .
$$

For the particular case $A+b=0$ the decomposition is different. We have

$$
P=\frac{1+c^{2}}{c^{2}} \frac{\partial\left(V_{1} V_{2}\right)}{\partial y}+P_{1} V_{2}+P_{2} V_{1},
$$

$$
Q=-\frac{1+c^{2}}{c^{2}} \frac{\partial\left(V_{1} V_{2}\right)}{\partial x}+Q_{1} V_{2}+Q_{2} V_{1},
$$

where $c \neq 0$ and

(31)

$$
\begin{aligned}
& \left(P_{1}, Q_{1}\right)=(b, c), \quad \text { with } \quad V_{1}=1-b y+c x \quad \text { and } \\
& \left(P_{2}, Q_{2}\right)=\left(\frac{3 b+2 b c^{2}-b c x-3 b^{2} y-c^{2} y-2 b^{2} c^{2} y}{c^{2}}, \frac{1-b y}{c}\right),
\end{aligned}
$$

with

$$
V_{2}=(1-b y)^{2} .
$$

The case $A+b=c=0$ is a particular case of (ii).

Finally, for condition (iv) we find the decomposition

$$
P=\frac{\partial\left(V_{1} V_{2}\right)}{\partial y}+P_{1} V_{2}+P_{2} V_{1},
$$

$$
Q=-\frac{\partial\left(V_{1} V_{2}\right)}{\partial x}+Q_{1} V_{2}+Q_{2} V_{1},
$$


with $a d \neq 0$ and

$$
\begin{aligned}
\left(P_{1}, Q_{1}\right)= & \left(-\frac{\left(1+2 a^{4} d^{2}+2 a^{2} d^{4}\right)}{a^{2} d}(1-a x+d y),\right. \\
& \left.-\frac{\left(1+2 a^{4} d^{2}+2 a^{2} d^{4}\right)}{2 a d^{2}}(a x-d y)\right),
\end{aligned}
$$

with

$$
\begin{aligned}
V_{1}= & d^{2}+a^{2}\left(a^{2}+d^{2}\right) x^{2}+2 d\left(a^{2}+d^{2}\right) y \\
& -2 a d\left(a^{2}+d^{2}\right) x y+d^{2}\left(a^{2}+d^{2}\right) y^{2} \quad \text { and } \\
\left(P_{2}, Q_{2}\right)= & \left(\frac{\left(1-3 a^{4} d^{2}-3 a^{2} d^{4}\right)}{a^{2} d}\left(1-a x+a^{2} x^{2}+2 d y-2 a d x y+d^{2} y^{2}\right),\right. \\
& \left.\frac{\left(1-3 a^{4} d^{2}-3 a^{2} d^{4}\right)}{a d^{2}}\left(a^{2} x^{2}+d y-2 a d x y+d^{2} y^{2}\right)\right),
\end{aligned}
$$

with

$$
\begin{aligned}
V_{2}(x, y)= & d^{2}-a^{3}\left(a^{2}+d^{2}\right) x^{3}+3 d\left(a^{2}+d^{2}\right) y \\
& -3 a d\left(a^{2}+d^{2}\right) x y+3 a^{2} d\left(a^{2}+d^{2}\right) x^{2} y+3 d^{2}\left(a^{2}+d^{2}\right) y^{2} \\
& -3 a d^{2}\left(a^{2}+d^{2}\right) x y^{2}+d^{3}\left(a^{2}+d^{2}\right) y^{3} .
\end{aligned}
$$

The case $a d=0$ is a particular case of condition (ii).

In all cases, the vector fields used in the decomposition of (24) are Hamiltonian or linear systems.

For the four examples that we have shown above, we have decomposed systems studied by several authors, in terms of more simple vector fields, by using expression (8).

\section{Systems with the same null divergence factor}

If two systems have the same null divergence factor, we can construct a more general system which has such null divergence factor, as it is shown in the following proposition: 
Proposition 2. Let $\left(P_{1}, Q_{1}\right)$ and $\left(P_{2}, Q_{2}\right)$ be two $C^{1}$ vector fields defined in an open subset $U \subset R^{2}$, which have the same null divergence factor $V(x, y)$, i.e.

$$
\begin{aligned}
& P_{1} \frac{\partial V}{\partial x}+Q_{1} \frac{\partial V}{\partial y}-\left(\frac{\partial P_{1}}{\partial x}+\frac{\partial Q_{1}}{\partial y}\right) V=0 \\
& P_{2} \frac{\partial V}{\partial x}+Q_{2} \frac{\partial V}{\partial y}-\left(\frac{\partial P_{2}}{\partial x}+\frac{\partial Q_{2}}{\partial y}\right) V=0
\end{aligned}
$$

then the vector field $\left(P_{1}+\lambda P_{2}, Q_{1}+\lambda Q_{2}\right)$ has also the function $V(x, y)$ as a null divergence factor, for arbitrary values of the parameter $\lambda$.

Proof: It is obvious from the definition of the null divergence factor.

Let us consider several examples in order to illustrate the utility of this proposition.

Example 1. In [3] we have studied the following cubic system:

$$
\begin{aligned}
& P=\lambda x-y+\lambda m_{1} x^{3}+\left(m_{2}-m_{1}+m_{1} m_{2}\right) x^{2} y+\lambda m_{1} m_{2} x y^{2}+m_{2} y^{3} \\
& Q=x+\lambda y-x^{3}+\lambda m_{1} x^{2} y+\left(m_{1} m_{2}-m_{1}-1\right) x y^{2}+\lambda m_{1} m_{2} y^{3}
\end{aligned}
$$

where $\lambda, m_{1}$ and $m_{2}$ are arbitrary parameters.

This system presents a very rich behaviour, with a great number of bifurcations when the parameters $\lambda, m_{1}$ and $m_{2}$ are varied.

In $[\mathbf{3}]$ we have been able to study in an exact way all these bifurcations, from the null divergence factor of the system, given by

$$
V(x, y)=\left(x^{2}+y^{2}\right)\left(1+m_{1} x^{2}+m_{1} m_{2} y^{2}\right) .
$$

Using Proposition 2, we can write system (35) as the composition of two more simple cubic systems

$$
\begin{aligned}
& P=P_{1}+\lambda P_{2}, \\
& Q=Q_{1}+\lambda Q_{2},
\end{aligned}
$$

where

$$
\begin{aligned}
\left(P_{1}, Q_{1}\right)= & \left(y\left(-1+m_{2} y^{2}+\left(m_{2}-m_{1}+m_{1} m_{2}\right) x^{2}\right)\right. \\
& \left.x\left(1-x^{2}-y^{2}-m_{1} y^{2}+m_{1} m_{2} y^{2}\right)\right) \quad \text { and } \\
\left(P_{2}, Q_{2}\right)= & \left(x\left(1+m_{1} x^{2}+m_{1} m_{2} y^{2}\right), y\left(1+m_{1} x^{2}+m_{1} m_{2} y^{2}\right)\right) .
\end{aligned}
$$

These two systems have the same null divergence factor, given by (36). 
System $\left(P_{1}, Q_{1}\right)$ has a center at the origin, and system $\left(P_{2}, Q_{2}\right)$ has a curve of critical points, given by $1+m_{1} x^{2}+m_{1} m_{2} y^{2}=0$.

These two systems have a simple qualitative behaviour, but their combination given by (37) presents a very complex pattern of bifurcations.

Example 2. Let us consider the two vector fields

$$
\begin{aligned}
& \left(P_{1}, Q_{1}\right)=\left(-y-b x^{2}-d y^{2}, x+A x y\right) \text { and } \\
& \left(P_{2}, Q_{2}\right)=\left(V_{1}(x, y), V_{1}(x, y)\right)
\end{aligned}
$$

where $V_{1}(x, y)$ is the null divergence factor of $\left(P_{1}, Q_{1}\right)$, given by:

$$
V_{1}(x, y)=(1+A y) W(x, y)
$$

where

$$
\begin{aligned}
W(x, y)= & -A-b+d+b(A+b)(A+2 b) x^{2} \\
& +2 b(A+b-d) y+b d(A+2 b) y^{2} .
\end{aligned}
$$

The vector field $\left(P_{1}, Q_{1}\right)$ is a quadratic integrable system with a center at the origin. The system $\left(P_{2}, Q_{2}\right)$ is a trivial vector field, which has also a null divergence factor given by (40). From Proposition 2 we can generate a cubic system

$$
\begin{aligned}
& P=-y-b x^{2}-d y^{2}+\lambda V_{1}(x, y), \\
& Q=x+A x y+\lambda V_{1}(x, y),
\end{aligned}
$$

which has also a null divergence factor given by (40).

Example 3. Let us consider the two vector fields

$$
\begin{aligned}
& \left(P_{1}, Q_{1}\right)=\left(\frac{\partial H(x, y)}{\partial y}, \frac{-\partial H(x, y)}{\partial x}\right) \text { and } \\
& \left(P_{2}, Q_{2}\right)=(x f(x, y), y f(x, y)),
\end{aligned}
$$

where $H(x, y)=\left(x^{2}+y^{2}\right) f(x, y)$ and $f(x, y)$ is an arbitrary $C^{1}$ function.

These two systems have the same null divergence factor, given by:

$$
V(x, y)=\left(x^{2}+y^{2}\right) f(x, y) .
$$

Using Proposition 2 we obtain a new vector field $(P, Q)$

$$
\begin{aligned}
& P=\frac{\partial H}{\partial y}+\lambda x f(x, y), \\
& Q=-\frac{\partial H}{\partial x}+\lambda y f(x, y),
\end{aligned}
$$


with a null divergence factor given by (44).

The possible limit cycles of (45) must be contained in the set defined by the condition $f(x, y)=0$.

The problem can be in how to find vector fields $X=\left(P_{1}, Q_{1}\right)$ and $Y=\left(P_{2}, Q_{2}\right)$, such that both vector fields have the same null divergence factor.

An answer to this question is contained in the following proposition:

Proposition 3. Let $X=\left(P_{1}, Q_{1}\right)$ and $Y=\left(P_{2}, Q_{2}\right)$ be two $C^{1}$ vector fields defined in an open subset $U \subset R^{2}$. Assume that the local flows defined by the solutions of $X$ and $Y$ commute in the sense of Lie's bracket, that is $[X, Y]=0$, then the function $V=P_{1} Q_{2}-P_{2} Q_{1}$ is a null divergence factor for both systems.

Proof: Condition $[X, Y]=0$ is equivalent to

$$
\begin{gathered}
P_{1} \frac{\partial P_{2}}{\partial x}-P_{2} \frac{\partial P_{1}}{\partial x}+Q_{1} \frac{\partial P_{2}}{\partial y}-Q_{2} \frac{\partial P_{1}}{\partial y}=0 \\
P_{1} \frac{\partial Q_{2}}{\partial x}-P_{2} \frac{\partial Q_{1}}{\partial x}+Q_{1} \frac{\partial Q_{2}}{\partial y}-Q_{2} \frac{\partial Q_{1}}{\partial y}=0 .
\end{gathered}
$$

From these two equations it is easy to show that $V=P_{1} Q_{2}-P_{2} Q_{1}$ satisfies

$$
\begin{aligned}
& P_{1} \frac{\partial V}{\partial x}+Q_{1} \frac{\partial V}{\partial y}=\left(\frac{\partial P_{1}}{\partial x}+\frac{\partial Q_{1}}{\partial y}\right) V \\
& P_{2} \frac{\partial V}{\partial x}+Q_{2} \frac{\partial V}{\partial y}=\left(\frac{\partial P_{2}}{\partial x}+\frac{\partial Q_{2}}{\partial y}\right) V
\end{aligned}
$$

Commuting vector fields have been studied by several authors (see for instance [15], [16] and references therein). In these works, commuting systems have been studied in relation to the problem of isochronous centers, and the transversality condition $X \wedge Y=P_{1} Q_{2}-Q_{1} P_{2}=V \neq 0$ has been imposed. 
In this case, the commuting systems can not have limit cycles (the limit cycles are contained in the set defined by $V=0$, see $[\mathbf{9}]$ ).

However, if the transversality condition is not imposed, one of the two commuting systems can have limit cycles, as can be seen in the following example:

$$
\begin{aligned}
& \left(P_{1}, Q_{1}\right)=\left(-y+x\left(1-x^{2}-y^{2}\right), x+y\left(1-x^{2}-y^{2}\right)\right) \quad \text { and } \\
& \left(P_{2}, Q_{2}\right)=(-y, x) .
\end{aligned}
$$

The null divergence factor for both systems is

$$
V(x, y)=\left(x^{2}+y^{2}\right)\left(1-x^{2}-y^{2}\right) .
$$

These two systems commute in $R^{2}$. The vector field $\left(P_{1}, Q_{1}\right)$ has a unique limit cycle given by $x^{2}+y^{2}-1=0$. The vector field $\left(P_{2}, Q_{2}\right)$ has a global center. The curve $x^{2}+y^{2}-1=0$ is a trajectory for both systems. For one of these systems, this curve is a limit cycle, while for the other one it is one closed curve of the center.

In conclusion, commuting systems can have limit cycles. They can be easily determined from the equation

$$
V=P_{1} Q_{2}-P_{2} Q_{1}=0
$$

\section{References}

1. J. Chavarriga, Integrable systems in the plane with a center type linear part, Appl. Math. (Warsaw) 22 (1994), 285-309.

2. J. Chavarriga, A class of integrable polynomial vector fields, Appl. Math. (Warsaw) 23 (1995), 339-350.

3. J. Chavarriga, H. Giacomini and J. Giné, On a new type of bifurcation of limit cycles for a planar cubic system, Nonlinear Anal. (1997) (to appear).

4. J. Chavarriga And J. Giné, Integrability of a linear center perturbed by fourth degree homogeneous polynomials, Publ. Mat. 40 (1996), 21-39. 
5. J. Chavarriga and J. Giné, Integrability of a linear center perturbed by fifth degree homogeneous polynomials, to appear in Publ. Mat. 41 (1997).

6. C. Christopher, Invariant algebraic curves and conditions for a center, Proc. Roy. Soc. Edinburgh Sect. A 124 (1994), 1209-1229.

7. C. Christopher and N. Lloyd, On the paper of Jin and Wang concerning the conditions for a center in certain cubic systems, Bull. London Math. Soc. 22 (1990), 5-12.

8. G. Darboux, Mèmoire sur les équations différentielles algebraiques du premier ordre et du premier degré, Bull. Sci. Math. (2) 2(1) (1878), 60-96;123-144;151-200.

9. H. Giacomini, J. Llibre and M. Viano, On the nonexistence, existence and uniqueness of limit cycles, Nonlinearity 9 (1996), 501516.

10. H. Giacomini, J. Llibre and M. Viano, On the shape of limit cycles that bifurcate from Hamiltonian centers, Preprint, Université de Tours, France (1997), submitted to Nonlinearity.

11. H. Giacomini and M. Ndiaye, New sufficient conditions for a center and global phase portraits for polynomial systems, Publ. Mat. 40 (1996), 351-372.

12. H. Giacomini and M. Viano, Determination of limit cycles for two-dimensional dynamical systems, Phys. Rev. E 52 (1995), 222228.

13. R. Kooij And C. Christopher, Algebraic invariant curves and the integrability of polynomial systems, Appl. Math. Lett. 6 (1993), $51-53$.

14. R. Kooij And A. Zegeling, Polynomial systems with centers and limit cycles, Reports of the Faculty of Technical Mathematics and Informatics 92-58 (1992), Delft, University of Technology.

15. M. Sabatini, Characterizing Isochronous Centres by Lie Brackets, to appear in Differential Equations Dynam. Systems.

16. M. Sabatini, Dynamics of commuting systems on two-dimensional manifolds, to appear in Ann. Mat. Pura Appl. 
1991 Mathematics subject classifications: Primary 34C05; Secondary 34A05.

\author{
J. Chavarriga: \\ Departament de Matemàtica \\ Universitat de Lleida \\ Plaça Victor Siurana 1 \\ 25003 Lleida \\ SPAIN \\ e-mail: chava@eup.udl.es
}

\author{
H. Giacomini: \\ Laboratoire de Mathématiques \\ et Physique Théorique \\ CNRS UPRES A6083 \\ Faculté des Sciences et Techniques \\ Parc de Grandmont \\ 37200 Tours \\ FRANCE
}

e-mail: giacomini@univ.tours.fr

\author{
J. Giné: \\ Departament de Matemàtica \\ Universitat de Lleida \\ Plaça Victor Siurana 1 \\ 25003 Lleida \\ SPAIN \\ e-mail: gine@eup.udl.es
}

Primera versió rebuda el 30 de Novembre de 1996, darrera versió rebuda el 8 d'Abril de 1997 